\title{
Okadaic acid depuration in the mussel Mytilus galloprovincialis: one- and two-compartment models and the effect of environmental conditions
}

\author{
Juan Blanco ${ }^{1, *}$, María Luisa Fernández ${ }^{2}$, Aurea Míguez ${ }^{2}$, Ángeles Moroño ${ }^{1}$ \\ ${ }^{1}$ Centro de Investigacións Marin̄as, Consellería de Pesca, Marisqueo e Acuicultura, Xunta de Galicia, Pedras de Corón s/n, \\ Aptdo. 13, E-36620 Vilanova de Arousa, Spain \\ ${ }^{2}$ Laboratorio de Sanidad Exterior de Vigo, European Community Reference Laboratory on Marine Biotoxins, \\ Estación Marítima s/n, E-36271 Vigo, Spain
}

\begin{abstract}
Following an episode of Diarrhetic Shellfish Poisoning (DSP) in mussels Mytilus galloprovincialis from the Ría de Pontevedra (Galicia, NW Spain), produced by organisms of the genus Dinophysis (dinoflagellate), a batch of contaminated mussels was transplanted to several uncontaminated sites having different environmental conditions. The depuration kinetics of okadaic acid (the dominant toxin involved in the episodel in each experimental mussel batch was monitored during a $70 \mathrm{~d}$ period, as were the corresponding salinity, temperature, fluorescence, light transmission and mussel body weight. Environmental conditions and body weight were shown to have an effect on depuration rates by principal component and multiple regression analysis. Although most of the variables studied showed a high degree of covariation, fluorescence and light transmission, which serve to measure the availability of solids and phytoplankton for mussels, appeared to have the most prominent effect on depuration. Four different models were tested in order to describe depuration kinetics: 1 - and 2 -compartment models, each in 2 versions, with and without the inclusion of the effects of the 4 environmental variables studied and body weight. The 1-compartment model without the complementary variables did not give an appropriate description of the kinetics, but when these variables were included, the general fitting was good and a pattern was found in the most important deviations. The simplest of the 2-compartment models fitted also produced an output that adequately described the kinetics observed. However, attempts to estimate the parameters of 2 -compartment models including the complementary variables consistently produced a 1 -compartment model. The model chosen to describe depuration kinetics substantially influences the estimation of the effect of the environmental variables
\end{abstract}

KEY WORDS: Okadaic acid Depuration - Models Mussel - Salinity - Temperature - Body weight Fluorescence Light transmission

\section{INTRODUCTION}

Marine phycotoxins are becoming one of the major threats to aquaculture and particularly to bivalve cultures (Shumway \& Cembella 1993). There is a wide diversity of phycotoxins that differ in chemical structure and properties as well as in their biological activity. Their repercussions on aquaculture vary both geo-

•E-mail: jblanco@cimacoron.org graphically and temporally. Among the biologically active compounds, DSP (Diarrhetic Shellfish Poisoning) toxins are one of the most important types, because they are widely distributed and their episodes are usually long-lasting. The main toxins in this group are okadaic acid, DTX-1 (Murata et al. 1982) and DTX2 (Hu et al. 1992, Blanco et al. 1995, Gago et al. 1996) and their acyl-derivatives, formerly described as DTX-3 (Yasumoto et al. 1985, Marr et al. 1992, Fernández et al. 1996). These toxins have diarrheagenic effects (Yasumoto et al. 1978) and are tumor promoters 
(Fujiki et al. 1988, Haystead et al. 1989, Suganuma et al. 1989). Even though these toxins do not cause mortality, morbidity is very high, and their presence in very small concentrations in bivalves makes them unsafe for human consumption. In most countries in which bivalve aquaculture is economically important, prolonged periods of shellfish contamination have led to great economic losses due to market bans.

Several approaches can be used to reduce the impact of DSP episodes. One is to reduce the importance of the toxic episode itself by reducing the contamination level and/or the duration of the depuration period, mainly by means of culture manipulation. Another possibility is to find a better management strategy for the culture by means of predicting the most likely duration of the episodes. Knowledge of both depuration kinetics and the effect of environmental conditions on depuration is essential to any of these approaches.

The depuration of several species of shellfish previously affected by DSP episodes has been studied by different authors. In some studies (Marcaillou-Le Baut et al. 1993, Shumway \& Cembella 1993), a 2-step depuration curve was found, with high depuration rates during the first few days and much smaller rates after. wards. In other cases (Croci et al. 1994), a unique depuration rate appears to exist for the whole depuration period. As in the case of PSP (Paralytic Shellfish Poisoning) toxins, these 2 responses can be described by 2 - and 1 -compartment models, respectively (Silvert \& Cembella 1995, Blanco et al. 1997). Blanco et al. (1995) have shown that a very simple 1-compartment

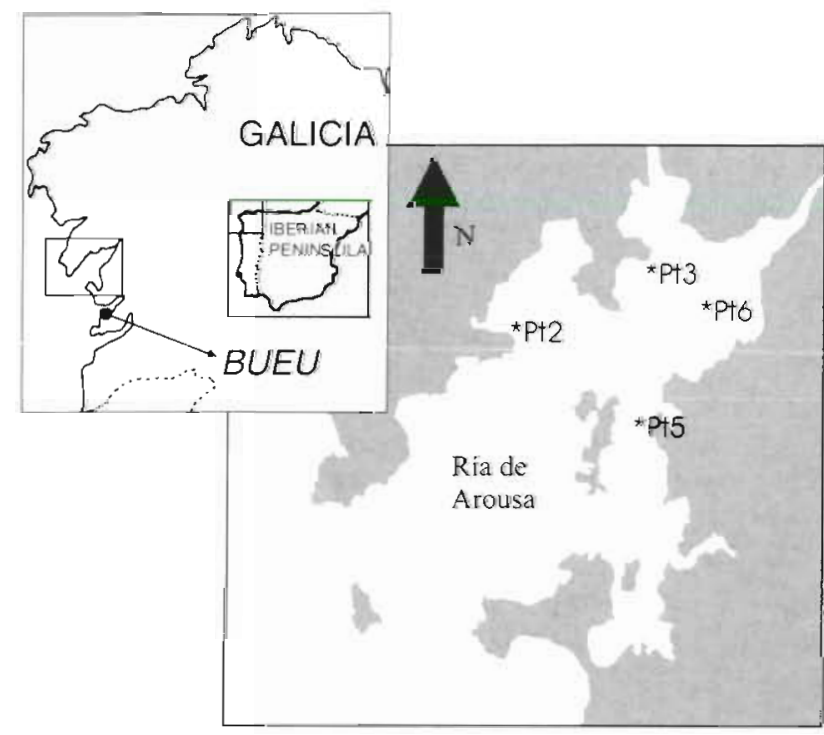

Fig. 1. Experimental sampling stations (Pt2, Pt3, Pt5 and Pt6) in the Ría de Arousa, Spain, and origin of the contaminated mussels Mytilus galloprovincialis (Ría de Pontevedra, Bueu) model can reasonably describe some DSP episodes in Galicia (Spain), but more complex models have not been tested.

The effect of the environmental conditions on depuration has not been directly evaluated. It is generally assumed that low temperatures lead to low depuration rates (Shumway \& Cembella 1993) but no strong evidence of this fact exists. Differences in depuration. rates between mussels maintained in a culture pond and at sea have also been shown (Marcaillou-Le Baut et al. 1993) and they have been attributed to differences in food resources.

The purpose of this work was to check the adequacy of 1-and 2-compartment models to describe the depuration kinetics of mussels Mytilus galloprovincialis affected by a Dinophysis acuminata and $D$. acuta episode in Galicia, and to study the effect of several environmental variables on the depuration rate.

\section{MATERIALS AND METHODS}

Experiment description and sample processing. In November 1994, mussels Mytilus galloprovincialis contaminated with DSP toxins (checked by HPLC, see 'Results') by a bloom of Dinophysis acuta and $D$. acuminata which had started in September and persisted on the date of sampling, were collected from Bueu (Ría de Pontevedra, Galicia, NW Spain; Fig. 1) and selected by shell length for an approximate size of $9 \mathrm{~cm}$ (mean $=9.057 \mathrm{~cm}$; $95 \%$ confidence interval for the individual sizes $=7.817$ to $10.297 \mathrm{~cm}$ ). Finally, the mussels were randomly distributed among culture baskets (60 mussels each) with walls of $1 \mathrm{~cm}$ mesh that were distributed, also randomly, among 4 rafts (Pt 2, Pt 3, Pt 5 and Pt 6; Fig. 1) with 2 depths each (3 and $8 \mathrm{~m}$ ), for a total of 8 sampling points, in the Ría de Arousa. Rafts and depths were chosen to minimize the covariation between salinity, temperature, fluorescence (as a measure of phytoplankton.) and light transmission (as a measure of total seston volume) and also to offer a low probability of re-contamination. The choice was based on the analysis of weekly data obtained by the Centro de Control da Calidade do Medio Mariño (Consellería de Pesca, Xunta de Galicia) for November and December of the 3 previous years. Re-contamination was avoided but the elimination of the covariation between variables was not achieved.

Mussels were sampled (15 individuals from every combination of sampling point and depth) on a weekly basis at first and, when the depuration slowed down, at variable intervals. Environmental conditions were sampled twice a week during most of the experiment 


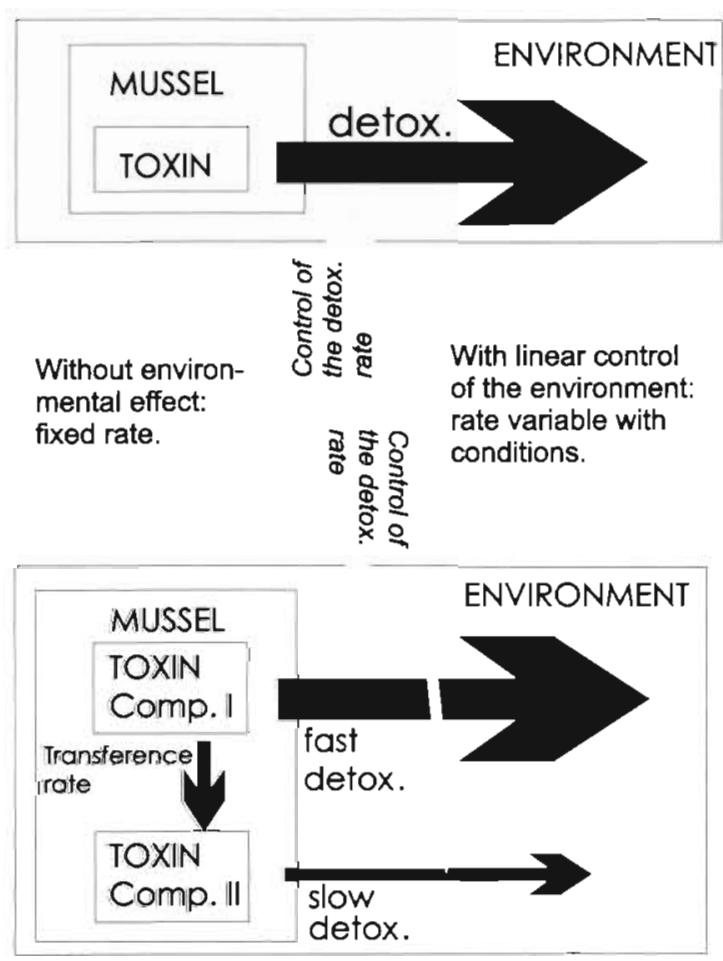

Fig. 2. Conceptual representation of the 1-compartment and 2-compartment models. Solid arrows represent toxin flows and shaded arrows represent the environmental control of the rates

with a CTD Sea-Bird 25 equipped with a Seatech Fluorometer and an integrated transmissometer

Once in the laboratory, mussels were measured and weighed, and the 15 digestive glands pooled and homogenized. The DSP toxins contained in the homogenate were extracted and analyzed following the procedure given by Lee et al. (1987).

The effect of the environmental conditions and body weight on the depuration rate was identified by Principal Component Analysis (PCA) and quantified by multiple regression with forward inclusion using the Minitab 11 Statistical Package. Fluorescence data were logarithmically transformed. Two types of regressions and PCAs were carried out: (1) general, using all data of the experiment (all depuration periods and all sampling points and depths) and (2) per depuration period, using the 8 sampling points from each individual sampling date. Unless otherwise noted all significant observations or comparisons had a significance level of $95 \%(p \leq 0.05)$.

Models. One- and two-compartment models, which have previously been used to describe PSP depuration kinetics (Blanco et al. 1997), were also used in this study (Fig. 2). In the 1-compartment model it was assumed that all toxicity in the digestive glands of the mussels has the same depuration kinetics. Two ver- sions of this basic model were used: one in which the depuration rate was independent from the environmental variables and body weight (it was therefore a constant), and another in which depuration rate was considered to be a linear combination of the environmental variables considered in this study and of the body weight (it was therefore calculated by a regression equation). The 2 versions of the 1 -compartment model are formally described as follows

Version 1 - constant depuration rate

$D R=K$

$\mathrm{d}($ TOX $) / \mathrm{d} t=-D R \cdot$ TOX

Version 2 - depuration rate linearly related with environmental variables and body weight

$D R=a+b \cdot S+c \cdot T+d \cdot F+e \cdot T_{I}+f \cdot B w$

$\mathrm{d}($ TOX $) / \mathrm{d} t=-D R \cdot$ TOX

where $D R=$ depuration rate $\left(\mathrm{d}^{-1}\right), a$ to $f$ are constants, Tox $=$ okadaic acid concentration in the digestive gland (expressed as $\mu \mathrm{g} \mathrm{g}^{-1}$ ), $S=$ salinity (psu), $T=$ temperature $\left({ }^{\circ} \mathrm{C}\right), F=$ fluorescence (volts), $T r=$ transmittance (percentage of light transmitted) and $B W=$ body weight $(g)$

In the 2-compartment model it is also assumed that toxins are distributed in 2 compartments, the first having a fast and the second a slow depuration rate, and that a transfer from the first to the second compartment exists. The same 2 versions as in the 1-compartment model (constant or dependent on the environment depuration rates) were used. The formulation of these 2-compartment models is as follows:

Version 1 - constant depuration rate

$D R_{1}=K_{1}$

$D R_{2}=K_{2}$

$\mathrm{d}\left(\operatorname{Tox}_{1}\right) / \mathrm{d} t=-D R_{1} \cdot$ Tox $_{1}-\mathrm{CR} \cdot$ Tox $_{1}$

$\mathrm{d}\left(\operatorname{Tox}_{2}\right) / \mathrm{d} t=-D R_{2} \cdot \operatorname{Tox}_{2}+\mathrm{CR} \cdot \operatorname{Tox}_{1}$

$\mathrm{d}(T O X) / \mathrm{d} t=\mathrm{d}\left(\right.$ TOX $\left._{1}\right) / \mathrm{d} t+\mathrm{d}\left(\right.$ Tox $\left._{2}\right) / \mathrm{d} t$

Version 2 -depuration rate linearly related with environmental variables and body weight

$D R_{1}=a_{1}+b_{1} \cdot S+c_{1} \cdot T+d_{1} \cdot F+e_{1} \cdot T r+f_{1} \cdot B W$

$D R_{2}=a_{2}+b_{2} \cdot S+c_{2} \cdot T+d_{2} \cdot F+e_{2} \cdot T r+f_{2} \cdot B w$

$\mathrm{d}\left(\right.$ Tox $\left._{1}\right) / \mathrm{d} t=-D R_{1} \cdot$ Tox $_{1}-\mathrm{CR} \cdot$ Tox $_{1}$

$\mathrm{d}\left(\operatorname{Tox}_{2}\right) / \mathrm{d} t=-D R_{2} \cdot \operatorname{Tox}_{2}+\mathrm{CR} \cdot \operatorname{Tox}_{1}$

$\mathrm{d}(T O X) / \mathrm{d} t=\mathrm{d}\left(T_{O X_{1}}\right) / \mathrm{d} t+\mathrm{d}\left(T o x_{2}\right) / \mathrm{d} t$

where $C R$ is the net transport rate from the first to the second compartment, numerical subscripts indicate compartment, and the remaining variables are as in the 1-compartment model.

The models which did not include environmental variables were fitted to the data by minimizing the sum of squares of the residuals by varying iteratively the depuration rate in the case of the 1-compartment model, or the depuration rates corresponding to each compartment, the transfer rate between compartments 
and the initial proportion of the toxin contents in the first compartment in the case of the 2-compartment model.

The effect of the environmental variables was included in the models by means of the regression equations that link these variables with the depuration rate. In the 1-compartment model, only 1 regression is needed but in the 2-compartment model 1 regression for each compartment has to be estimated: one for the fast depurating and another for the slow depurating compartment (Compartments 1 and 2 respectively). All data obtained were used for fitting the regression needed for the 1 -compartment model. For the 2-compartment model, all data corresponding to the first detoxification period were considered to be mostly affected by the fast depuration compartment (as depuration rates in this period are the fastest in the experiment) and they were, therefore, used to fit the first regression. The last 3 depuration periods were considered to be mainly affected by the slow depurating compartment and were, therefore, used to fit the second regression. The environmental effect was also directly implemented in the 2-compartment model by means of including the 2 regressions in the model and afterwards allowing their coefficients to vary during the iterative fitting process described previously.

\section{RESULTS}

\section{Environmental variables and body weight}

The environmental conditions showed differences in both time and space. The temperature range throughout the period studied was $2.2^{\circ} \mathrm{C}$, from approximately 13 to $15.2^{\circ} \mathrm{C}$. As is typical for a winter situation with thermal inversion, the surface had lower temperatures than the bottom. Salinity ranged from approximately 27 to 34.4 psu. Fluorescence and light transmission also showed great differences with both time and sampling point. Body weight also presented great differences and a slightly ascending general trend. Only for 2 out of the 8 sampling points was the final body weight lower than at the start, and these 2 points were also the ones with the greatest salinity oscillations during the period studied. A more detailed description of the environmental variables can be found in Blanco et al. (1997).

\section{Toxins, depuration rates and principal component} analysis

Okadaic acid was the only toxin detected in relevant concentrations $\left(4.93 \mu \mathrm{g} \mathrm{g}^{-1}\right.$ of digestive gland at the beginning of the experiment). Its depuration rate during this study ranged from 0.22 to $0.02 \mathrm{~d}^{-1}$ (Fig. 3). A decreasing trend for the first 4 depuration periods and stabilization from this point to the end of the experiment was observed.

The depuration curves observed showed an alternating pattern in which a low depuration rate followed a high one. This effect is probably due to the experimental error together with the calculation of the rates, since an observation that underestimates the real toxicity produces an overestimation of the previous rate and a subsequent underestimation of the following one. To partially avoid this problem we smoothed the data by means of averaging the observations corresponding to each 2 consecutive dates. We carried out the PCA and the regression analyses corresponding to the individual detoxification periods using these transformed data. In the analysis of the whole detoxification we used both smoothed and raw data.

Two groups of analysis were carried out. One included only the environmental variables and body weight, and the other also included the depuration rates. The first group (Figs. $4 \mathrm{~A}, \mathrm{C} \& 5 \mathrm{~F}$ to J) showed 2 principal components (PC) that account for most of the environmental and body weight variability. The envi- 

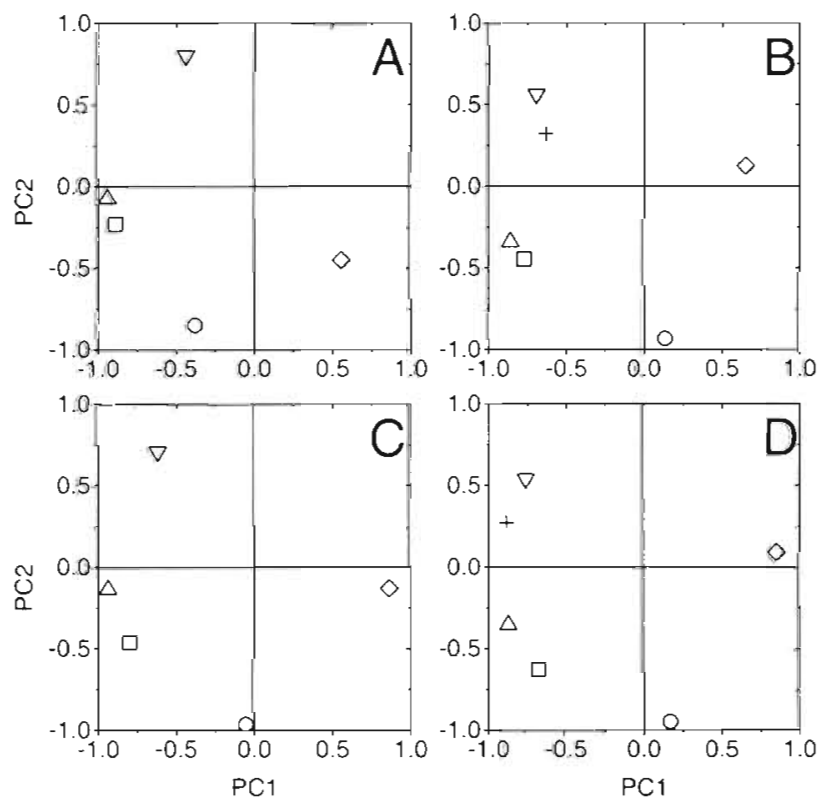

$\begin{array}{clccc}\square & \text { Temperature } 0 & \text { Salınity } \Delta & \Delta \text { Light Transmission } \\ \nabla & \text { Log Fluorescence } & \diamond & \text { Body Weight }+ \text { Detox. Rate }\end{array}$

Fig. 4. Mytilus galloprovincialis. Loadings of the environmental variables and fresh body weight $(A, C)$ and of the same variables also including okadaic acid depuration rate $(B, D)$ on the first 2 principal components ( $\mathrm{PC1}$ and $\mathrm{PC} 2$ ) extracted from the raw data $(A, B)$ and transformed by averaging each 2 consecutive sampling periods $(C, D)$ ronmental variables, with the exception of the global analysis of the experiment, were linked to 1 of the 2 extracted PCs (PC1 in most cases) and most of the body weight variability to the other. Salinity, temperature and light transmission were directly related to the PC that explained most of their variance, while fluorescence was inversely related. These 2 components accounted for most of the variation of the variables included in this study ( 82 to $89 \%$ of the variance).

The second group of analyses showed how the variability of the depuration rates related to the common variation of the environmental variables and body weight (Figs. 4B, D \& 5A to E). The inclusion of the depuration rates as a new variable in the analyses did not substantially affect the principal components extracted, meaning that most of its variability was linked to that of the environmental variables and body weight. The depuration rate was, in general, related to a PC characterized by a high positive loading on temperature, salinity and light transmission, and a negative loading on fluorescence. This component was PC1 in the analyses carried out with each individual depuration period (Fig. 5A to E) and PC2 in the global analysis of the experiment (Fig. 4B, D). In the cases where the depuration rate was not very well correlated with this component, it was related to the other PC extracted, which, in general, included the body weight, but was inversely related to the depuration rate. As in the previous group, most of the variability
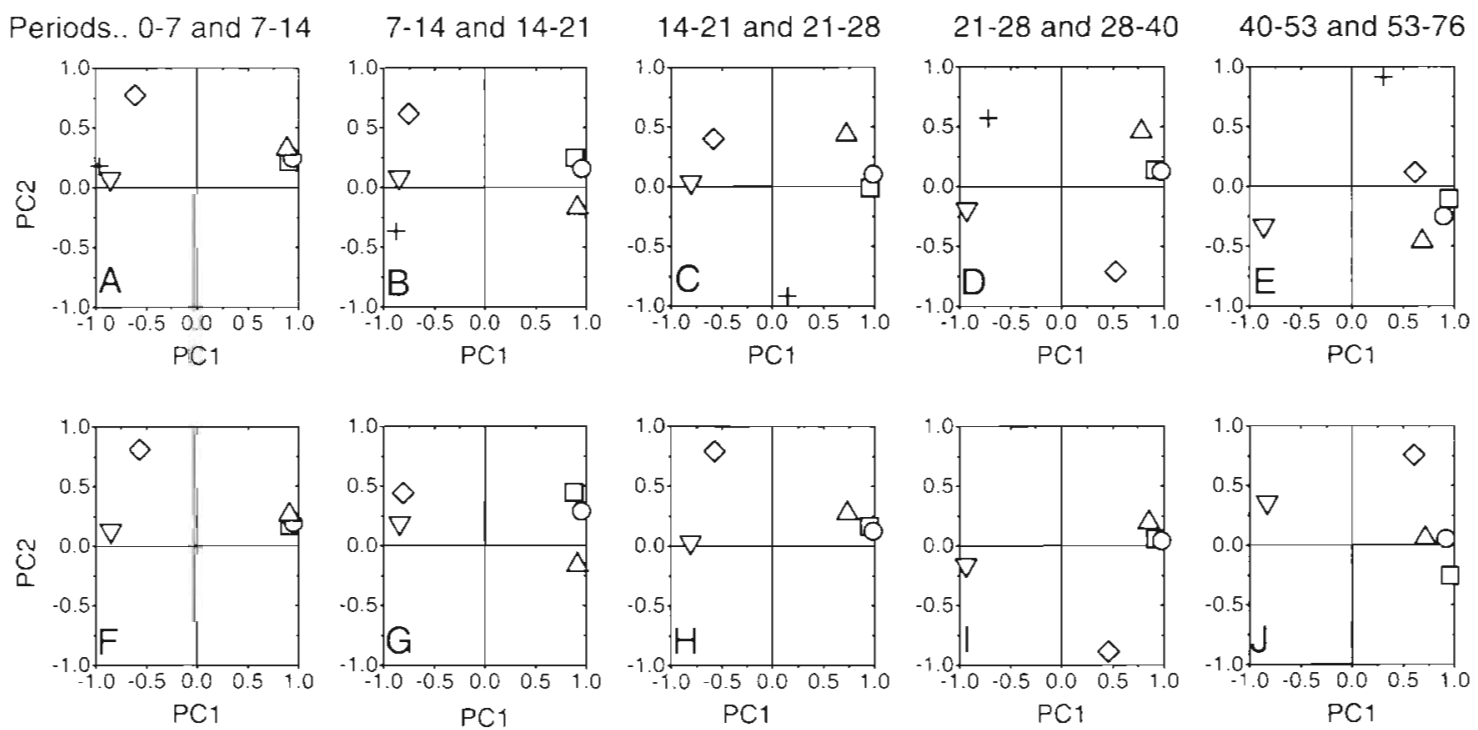

Temperature

0 Salinity $\Delta$ Light Transmission $\nabla$ Log Fluorescence

Body Weight

Fig. 5. Mytilus galloprovincialis. Loadings of the environmental variables and fresh body weight, including (A to E) and not including ( $F$ to J) okadaic acid depuration rate, on the first 2 principal components (PC1 and PC2) extracted from the data transformed by averaging each 2 consecutive sampling periods of the experiment. The averaged depuration periods (Days 0 to 7,7 to 14, etc.) appear above the corresponding plots 

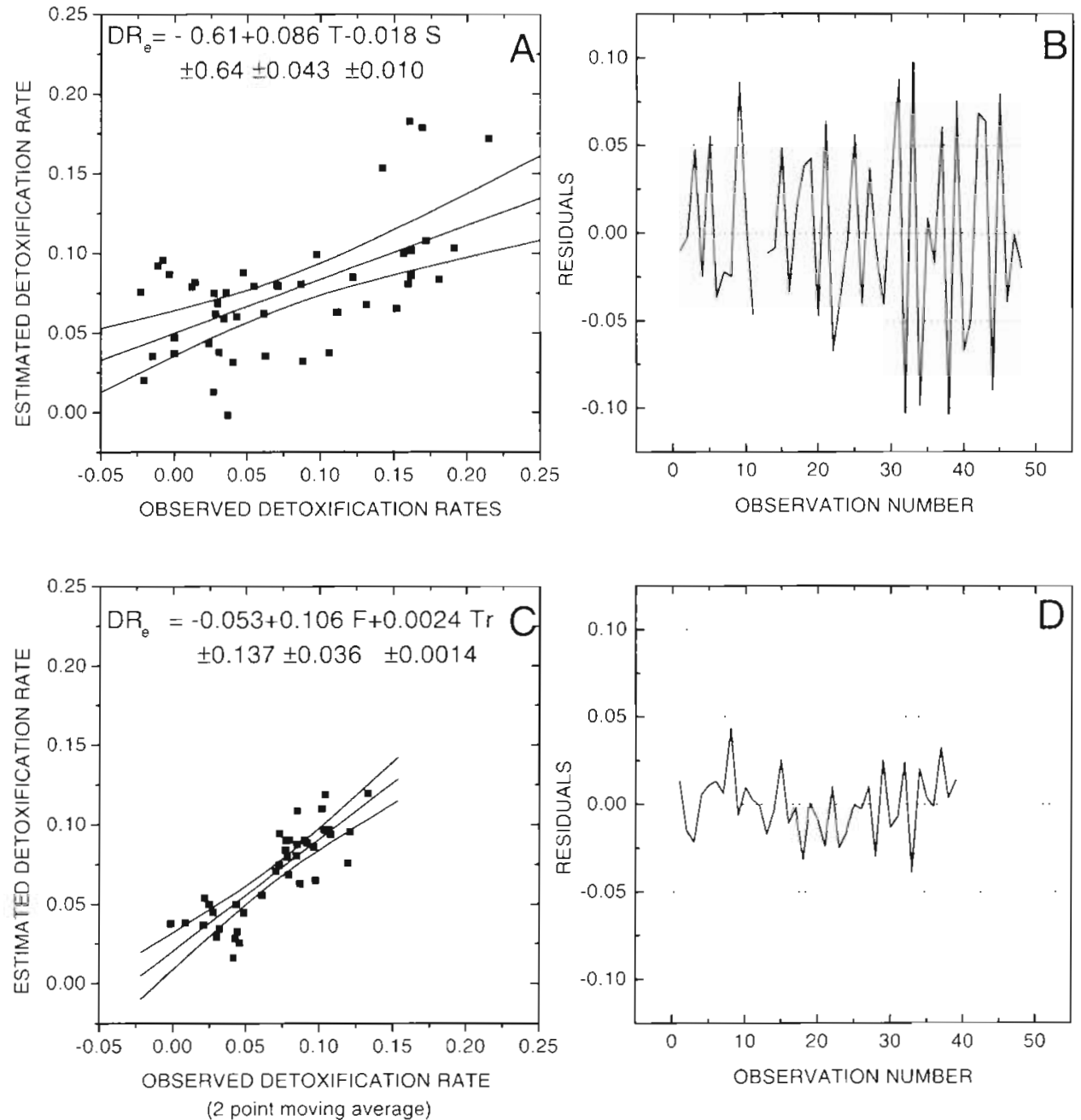

Fig. 6. Mytilus galloprovincialis. Results of the forward inclusion multiple regression analysis of okadaic acid depuration rate with the environmental variables and mussel fresh body weight. $T=$ temperature, $S=$ salinity, $F=$ fluorescence (logarithmically transformed) and $\mathrm{Tr}=$ light transmission. 95\% confidence limits for each coefficient and intercept are given below their corresponding values. (A) and (C) Relationship between depuration observed and depuration predicted by regression (DR), using raw and 2-point moving averaged data, respectively. (B) and (D) Corresponding residual deviations for (A) and (C)

(from 77 to $89 \%$ of the total variance) was explained by the 2 PCs extracted.

\section{General regression analysis}

Salinity and temperature were the only variables significantly related to depuration rate by forward inclusion multiple regression (Fig. 6A) (with negative and positive coefficients, respectively). Individually, however, the logarithmically transformed fluorescence was the variable most correlated with the depuration rate. As most of the variation can be explained by $2 \mathrm{PCs}$, one closely related to temperature, light transmission and body weight, and the other to fluorescence and salinity (the latter has a negative loading, Fig. $4 \mathrm{~A}, \mathrm{C}$ ) it is impossible to discriminate between the effects of the individual variables. Temperature was the variable most correlated with the depuration rate among the 3 related to PC1 (temperature, light transmission and body weight). The effect of salinity, the variable most significantly related to depuration rate by regression among those related with $\mathrm{PC}$, has no apparent explanation. In view of this difficulty, we decided to check the possibility that the main factor responsible for this relationship was, in fact, fluorescence (the other variable related to $\mathrm{PC}$ ), which was also the variable that showed the highest correlation with the depuration rate when considered individually. When salinity was excluded from the analysis, the 2 significant variables were temperature and fluorescence, and the total explained variance was reduced by approximately 
$4 \%$. Both multiple regressions accounted for a small percentage of the total variance $(33.9$ and $28 \%$ in the first and second cases respectively), and the corresponding residuals were found to be strongly affected by the sampling date, showing an alternating pattern (Fig. 6B). As no relation was found between these residuals and the other factors studied, we attributed them to the errors in toxin quantification, inherent in the technique, that led to a situation whereby if one rate is overestimated the next one is underestimated. To partially avoid this problem, as in the case of PCA, we smoothed all the variables by means of a moving average of every 2 consecutive points. When the same kind of regression was fitted to the new data, light transmission and fluorescence were the only 2 significant contributors (Fig 6C), both with positive coefficients, accounting for $70.6 \%$ of the total variance. If light transmission was removed from the analysis, following the same criteria as previously with salinity, then temperature and fluorescence were the significant variables and the percentage of variance explained was reduced by only $1.4 \%$. In this case, the alternating pattern of the residuals with the sampling date was also found, but was much less pronounced than in the analysis of the raw data (Fig. 6D).

\section{Regression analysis for each sampling date}

It was not possible to find any consistent relationship between the depuration rate in each depuration period and the envirommental variables. In several periods, the depuration rate was not significantly related to any environmental variable and, in the cases where such a relationship was present, the variables involved and the signs of the corresponding coefficients showed no recognizable pattern (Table 1 ).

As noted in the previous section, the residuals of the regression showed an alternating pattern, and we used

Table 1. Results of the forward inclusion regression for each sampling. $\{+\}$ and $(-)$ : sign of the coefficients when the varnables were included in the regression is shown. ni: variables not included in the equation. $T$. temperature (" $C$ ), S: salinity (psu), $T_{r}$ : percentage of light transmitted, $F$ : fluorescence, $B w$ : body weight $(g)$

\begin{tabular}{|c|c|c|c|c|c|}
\hline Day & $T$ & $S$ & $T r$ & $\log F$ & $B w$ \\
\hline 1 & ni & ni & ni & ni & $\mathrm{ni}$ \\
\hline 2 & ni & - & ni & nj & + \\
\hline 3 & ni & ni & $\mathrm{ni}$ & - & ni \\
\hline 4 & ni & ni & ni. & ni & $\mathrm{ni}$ \\
\hline 5 & ni & ni & $\mathrm{ni}$ & ni & $n i$ \\
\hline 6 & ni & ni & ni & ni & ni \\
\hline 1 to 6 & + & - & $\mathrm{nl}$ & $\mathrm{ni}$ & $\mathrm{ni}$ \\
\hline
\end{tabular}

Table 2. Results of the forward inclusion regression for the combination of each 2 consecutive sampling dates (moving average of the raw values). Sign (+ or - ) of the coefficients when the variables (see Table 1 for definitions) were included in the regression is shown. ni: variables not included in the equation

\begin{tabular}{|c|c|c|c|c|c|}
\hline Day & $T$ & $S$ & $\operatorname{Tr}$ & $\log F$ & $B w$ \\
\hline $1+2$ & ni & - & $\mathrm{ni}$ & $\mathrm{ni}$ & + \\
\hline $2+3$ & ni & - & ni & $\mathrm{ni}$ & ni \\
\hline $3+4$ & ni & nj & ni & ni & ni \\
\hline $4+5$ & ni & - & ni & ni & ni \\
\hline $5+6$ & ni & ni. & ni & ni & $\mathrm{ni}$ \\
\hline 1 to 6 & ni & ni & + & + & $\mathrm{ni}$ \\
\hline
\end{tabular}

2 -point moving averages instead of raw data. Of the 5 periods obtained (1 period is lost in the calculation of the moving averages) 3 showed significant results (Table 2). The negative relationship of depuration rate with salinity was the most relevant result. This variable shares most of its variation with temperature, light transmission and fluorescence, and it is consistently related to $\mathrm{PC} 1$ in the $\mathrm{PCA}$ corresponding to each date (Fig. 5).

\section{Model fitting}

All models fit the observed depuration quantitatively well, explaining 97, 96 and $99 \%$ of the variance (1compartment, 1-compartment with environmental variables and 2-compartment, respectively). Notwithstanding, they differed in the qualitative aspects of the fitting, which is shown by the way in which the residuals are distributed (Fig. 7). The 1-compartment model without environmental account did not correctly describe the final part of the depuration either in toxin content, which was underestimated (positive residuals, Fig. $7 \mathrm{~A}$ ), or in trend, with the actual data showing a decreasing trend that was not observed in the response expected from the model (Fig. 8A). When the results from the global regression between the depuration rate and the environmental variables were included, the model fit the observed data qualitatively better (Figs. $7 \mathrm{~B} \& 8 \mathrm{~B}$ ), as is shown by the much more centered distribution of the residuals (Fig. 7B), with the only exception being those corresponding to Day 7 .

The 2-compartment model (without including the environmental variables and body weight) fit the actual data well (Figs. 7C \& 9) The values obtained for the first sampling period were much closer to the observed values than in the 2 previous models and the residuals were fairly well centered. The estimated parameters for this model were a depuration rate for the first and second compartments of 0.17 and $0.10 \mathrm{~d}^{-1}$, 


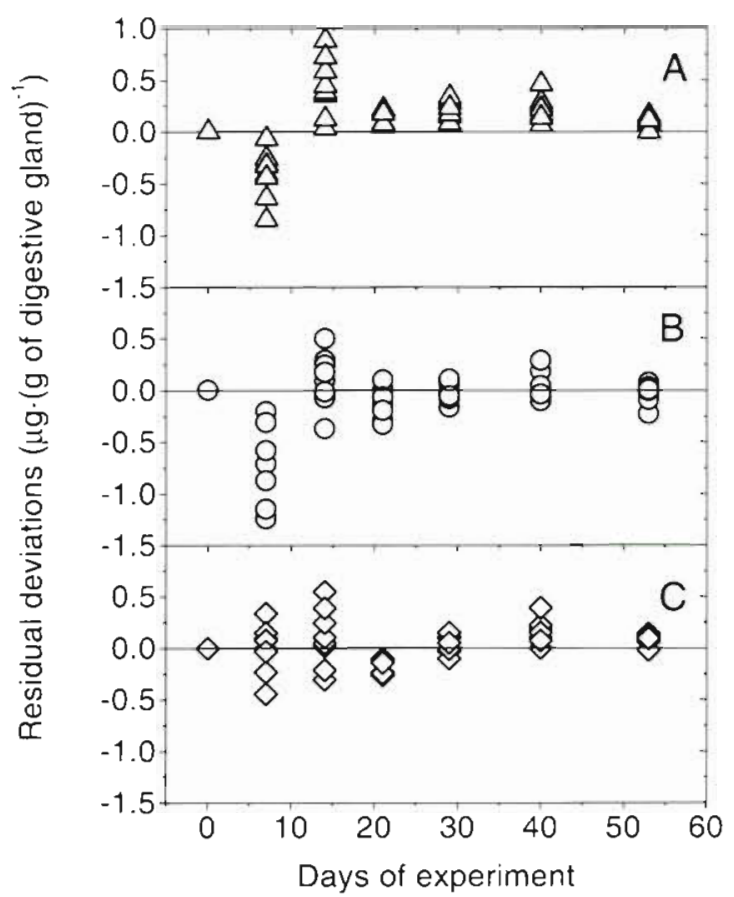

$\triangle$ One-compartment, independent of environment

One-compartment, rates depending on environment

$\diamond$ Two-compartment, independent of environment

Fig. 7. Mytilus galloprovincialis. Residual deviations corresponding to the 3 depuration models fitted to the observed data. (A) 1-compartment model independent of the environment, (B) 1-compartment model including the effect of environment, and (C) 2-compartment model independent of the environment respectively, a transfer rate between Compartments 1 and 2 of $0.31 \mathrm{~d}^{-1}$ and an initial contribution of toxins in the first compartment to the total of $99.9 \%$.

As mentioned in 'Materials and methods', we followed 2 different strategies to implement the effect of the environmental variables on the 2-compartment model. In both cases, after optimization, the parameters obtained caused the initial model to revert to a 1 compartment model because $100 \%$ of the initial toxicity was put in the first compartment and the transfer rate from Compartment 1 to Compartment 2 was estimated to be zero. This indicates that all the depuration takes place in Compartment 1 and, therefore, that the model is a 1-compartment model.

\section{DISCUSSION}

The depuration rates found in this study are very close to our previous estimates in the same area, although they were obtained by optimizing the fit of a contamination-depuration model (Blanco et al. 1995). They were slightly lower than those found in other studies on Mytilus galloprovincialis such as those by Poletti et al. (1996) and Croci et al. (1994) for depuration using ozone (from nearly 0 to $0.27 \mathrm{~d}^{-1}$ ). MarcaillouLe Baut et al. (1993) also found lower depuration rates, 0.07 and $0.08 \mathrm{~d}^{-1}$, for Mediterranean and Breton mussels respectively (in the latter case during the first $15 \mathrm{~d}$ and subsequently decreasing).

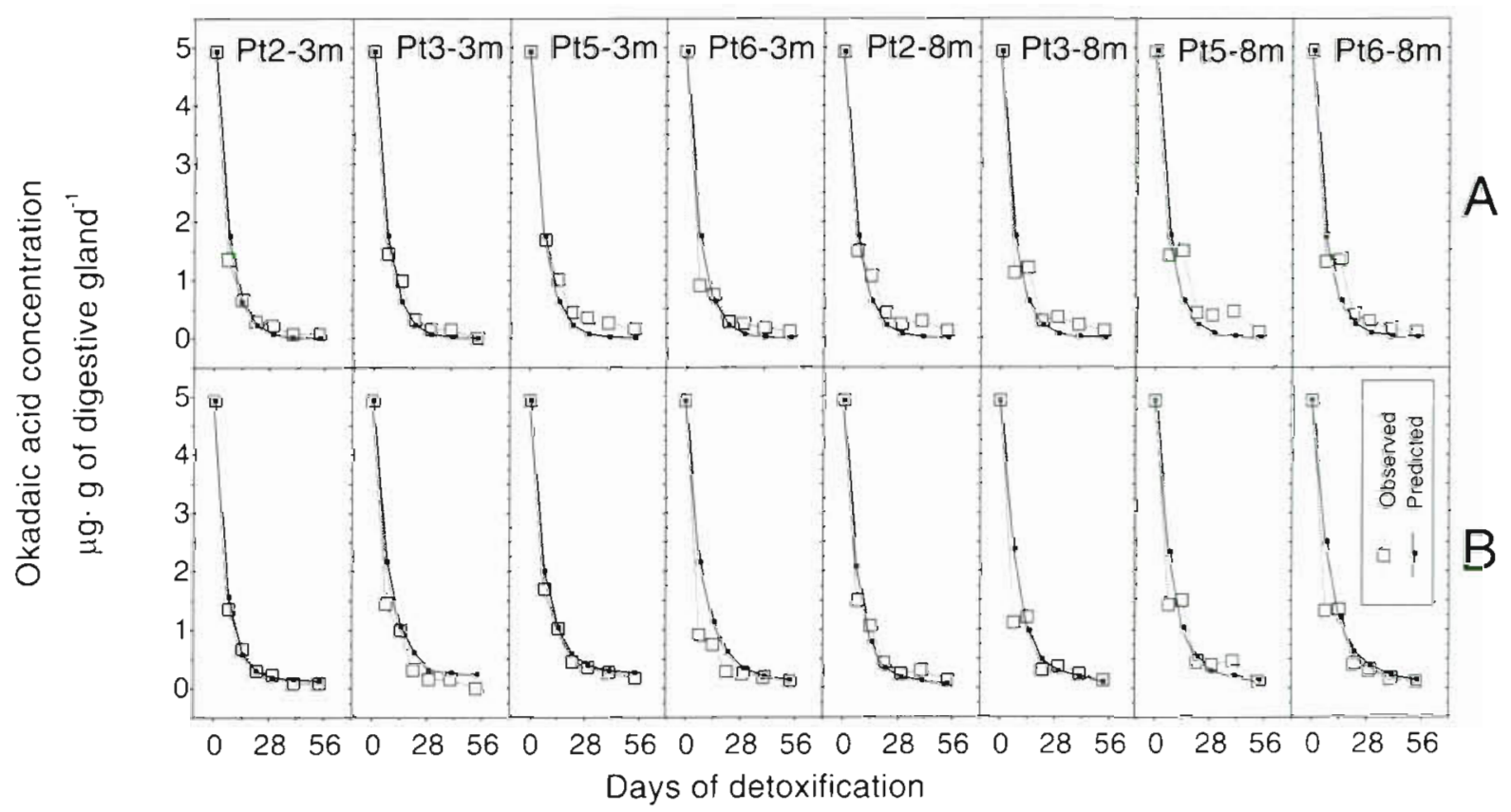

Fig. 8. Mytilus galloprovincialis. Depuration kinetics and fitted 1-compartment models for each sampling point (Pt2-3 m: Stn Pt 2 at $3 \mathrm{~m}$; Pt2-8 $\mathrm{m}$ : Stn Pt 2 at $8 \mathrm{~m}$; etc.) (A.) Fitted fixed rate models (without environmental effect) and (B) corresponding variable rate models (environmentally determined rates). Toxın concentration is expressed as $\mu g$ of total toxins per gram of digestive gland 


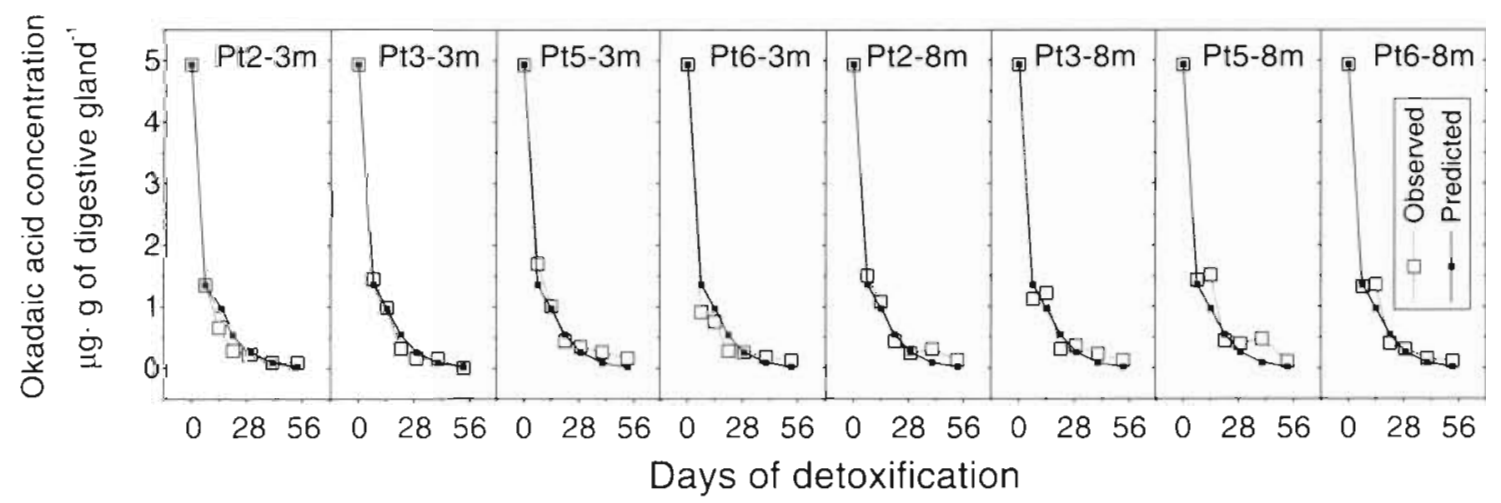

Fig. 9. Mytilus galloprovincialis. Depuration kinetics and fitted 2-compartment model for each sampling point. The fitted fixed rate model (without environmental effect) is shown. Toxin concentration is expressed as $\mu \mathrm{g}$ of total toxins per gram of digestive gland

From the PCA it would seem that, in general, the depuration rate was related to a group of variables that covary: temperature, salinity, light transmission and fluorescence; and also to body weight. Its inverse relationship with the PC in which temperature, light transmission and salinity had positive loadings and fluorescence negative, cannot be easily explained by temperature or salinity. Temperature, in the temperature range of this study, is expected to accelerate the metabolic processes and to increase the filtration rates in Mytilus edulis (Bayne et al. 1976, Jorgensen et al. 1990, Riisgård 1991, Hawkins \& Bayne 1992), and it would presumably have a similar effect on $M$. galloprovincialis. Those effects would probably lead to an increase in depuration rates rather than to the reduction found in this study. A negative effect of this factor on depuration therefore seems unlikely. The global regression analysis showed a positive relationship between these 2 variables, as would be expected, but the regressions obtained for each sampling date and the already described PCA indicated a negative relationship. The origin of this discrepancy seems to be the systematic decrease of temperature during the experiment. Due to this trend, the fastest depuration rates, which were characteristic of the early depuration period, were recorded simultaneously with the highest temperatures while the opposite situation was produced during later depuration. This trend did not affect the estimate of the effect of temperature when the individual depuration periods were analyzed and, therefore, in these individualized analyses, the actual effect of temperature and/or its covariates is estimated. Low salinity may have an effect, mainly through the decreasing of the osmotic pressure, but it also seems unlikely that it would affect the depuration of these kinds of compounds, which are lipid-soluble and which are highly likely to be bound to cellular components. Light transmission and fluorescence, however, may explain the effect of this group of variables. The former is inversely related to the seston concentration, in volume, and the latter to the phytoplankton concentration. The inverse relation of light transmission to depuration rate indicates that seston volume, and therefore the amount of food available for the mussels, may be a variable that actually affects the depuration process. The same conclusion may be drawn from fluorescence: its direct relationship with depuration rates also indicates that the phytoplankton concentration is involved in the regulation of the depuration rates. There is some evidence of the effect of phytoplankton concentration on the contamination-depuration kinetics of DSP toxins in mussels. The observations made by Sampayo et al. (1990) in several DSP episodes produced by Dinophysis spp. on the Portuguese coast suggest that depuration rates increase with phytoplankton concentration and also that, when the contribution of DSP producing species to the total phytoplankton biomass is low, the contamination of mussels is slower than when it is high. As in the case of PSP toxins (Blanco et al. 1997), we believe that phytoplankton concentration affects the process of depuration by increasing the digestive activity, which also leads to an increase in the metabolic fecal losses, thereby probably flushing out the bound toxins. These kinds of losses are made up mainly of remains of cells from the digestive gland tubules (Hawkins et al. 1990) which presumably had accumulated toxins and, in this way, are eliminated. As this mechanism may constitute as much as $60 \%$ of the organic matter in the feces (Hawkins et al. 1990), it might be the main elimination mechanism of the toxins contained in the digestive gland.

A relationship between depuration rate and body weight can also be extracted from the PCA. This relationship was inverse and not fully supported by the regression analysis, in which different responses were found on different dates. In some cases body weight 
seems to have a positive effect on depuration. These cases may be explained by the toxin dilution effect demonstrated for PSP toxins by Bricelj et al. (1990), and which is derived from the fact that, with constant toxin content, if the body weight of the bivalves increases, then the toxin concentration per unit of weight decreases and also by the fact that larger mussels have larger metabolic fecal losses (Hawkins et al. 1990). Nonetheless, in most of the cases in this experiment, there was an inverse relation between depuration and body weight, and therefore it cannot be explained as described previously. In our opinion, this relation was produced by the higher depuration capability of small mussels as compared to larger specimens, as was found for domoic acid (Wohlgeschaffen et al. 1992). The variation in mussel size due to the random sampling will probably allow for detection of this effect.

From our results it cannot be clearly concluded whether the DSP depuration in this species evolves following 1 - or 2-compartment kinetics because the benefit obtained by fitting a 2-compartment model is partially overlapped by the effect of including the environmental variables in the model. It is obvious that the 1-compartment model with a fixed depuration rate did not fit the actual data as well as the other 2 models, but the differences between the latter 2 models are not clear, with each having its strengths and its weaknesses. The 1-compartment model with environmental variables included fit the peculiarities of each data set better. The 2-compartment model, on the other hand, fit the first depuration point in each data set better. In our opinion the 1-compartment model that includes the environmental variables and body weight is more real. istic than the 2-compartment model because its main drawback, the poor fitting of the first point of each data set, (1) coincides with an atypically low okadaic acid concentration for these points followed by similar or higher toxin contents on the next sampling date, and (2) seems to be derived from one or several unknown factors which were not monitored during the experiment, as it showed a pattern in which the deviation of the model was larger in the bottom $(8 \mathrm{~m})$ than in the surface samples $(3 \mathrm{~m})$ and followed the same station order at each depth, and also because DSP analyses are carried out on the digestive gland, and therefore the second compartment does not involve other organs and, thus, might comprise a smaller fraction of the analyzed biomass than in the case of PSP (Blanco et al. 1997).

Our inability to fit the 2-compartment model including environmental conditions may also be affected by the precision of the toxin quantification of the first depuration point, because very small errors might make impossible the discrimination between 2 models that fit so well. If this point has been significantly affected by random sampling or analytical errors, our estimation of the effect on these conditions on the first compartment would be inadequate and the model might be incorrectly titted.

If we consider the 2-compartment model to be the most realistic, we must conclude that the environmental variables have little or no importance in regulating the depuration, which is in contrast with the small distortion that the inclusion of the depuration rate induced on the components extracted by PCA. In this case, the 2 compartments would have depuration rates which are very different from each other and a very high transfer rate $\left(0.3 \mathrm{~d}^{-1}\right)$.

In conclusion, it would appear that environmental conditions, mainly through the amount of available food, affect the depuration kinetics of okadaic acid, but the real influence of these variables cannot be quantified if a depuration model is not previously assumed.

Acknowledgements. This work was funded by the Consellería de Pesca, Marisqueo e Acuicultura, and CICYT (Project ALI-95-1012-C05-02). We thank M. C. Mariño and M Miranda for their technical assistence and the department of biotoxins of the Centro de Control de Calidade do Medio Marino for obtaining the experimental mussels and their help in the preparation of the experiment.

\section{LITERATURE CITED}

Bayne BL, Thompson. RJ, Widdows J (1976) Physiology I. In Bayne BL (ed) Marine mussels: their ecology and physiology. Cambridge University Press, Cambridge, p 121-206

Blanco J, Fernández M, Mariño J, Reguera B, Miguez A, Maneiro J, Cacho E, Martínez A (1995) From Dinophysis spp. toxicity to DSP outbreaks: a preliminary model of toxin accumulation in mussels. In.: Lassus P, Arzul G, Erard E, Gentien P, Marcaillou C (eds) Harmful marine algal blooms. Lavoisier, Intercept Ltd, Paris, p 777-782

Blanco J, Moroño A, Franco J, Reyero MI (1997) PSP depuration kinetics in the mussel Mytilus galloprovincialis. Oneand two-compartment models and the effect of some environmental variables. Mar Ecol Prog Ser 158:165-175

Bricelj VM, Lee JH, Cembella AD, Anderson DM (1990) Uptake and kinetics of paralytic shelltish toxins from the dinoflagellate Alexandrium fundyense in the mussel Mytilus edulis. Mar Ecol Prog Ser 63:177-188

Croci L, Toti L, De Medici D, Cozzi L (1994) Diarrhetic shellfish poison in mussels: comparison of methods of detection and determination of the effectiveness of depuration. Food Microbiol 24:337-342

Fernández ML, Míguez A, Cacho E, Martínez A (1996) Okadaic acid esters in Spanish mussels. Toxicon 34 : $381-387$

Fujiki $H$, Suganuma $M$, Suguri $H$, Yoshizawa S, Takagi $K$, Uda N, Wakamatsu K, Yamada K, Murata M (1988) Diarrhetic shellfish toxin dinophysistoxin-1 is a potent tumour promoter on mouse skin. Jpn J Cancer Res 79:1089-1.093

Gago A, Rodríguez Vázquez JA, Leao JM, Comesaña $M$, Quilliam MA, Thibault P (1996) 1985-1995. A decade of studies on PSP and DSP toxins in Spanish mussels by chemical methods. In: Yasumoto $T$, Oshima $Y$, Fukuyo $Y$ 
(eds) Harmful and toxic algal blooms. IOC of UNESCO, Paris, p $7-10$

Hawkins AJS, Bayne BL (1992) Physiological interrelations, and the regulation of production. In: Gossling $E$ (ed) The mussel Mytilus: ecology, physiology, genetics and culture. Elsevier, Amsterdam, p 171-222

Hawkins AJS, Navarro E, Iglesias JIP (1990) Comparative allometries of gut content, gut passage time and metabolic faecal loss in Mytilus edulis and Cerastoderma edule. Mar Biol 105:197-204

Haystead TAJ, Sim ATR, Carling D, Honnor RC, Tsukitani Y, Cohen P, Hardie DG (1989) Effect of the tumour promoter okadaic acid on intracellular protein phosphorylation and metabolism. Nature 337:78-81

Hu T, Doyke J, Jackson D, Marr J, Nixon E, Pleasance S, Quilliam MA, Walter JA, Wright JLC (1992) Isolation of a new diarrhetic shellfish poison from Irish mussels. Chem Commun 30:39-41

Jørgensen CB, Larsen PS, Riisgard HU (1990) Effects of temperature on the mussel pump. Mar Ecol Prog Ser 64:89-97

Lee JS, Yanagi T, Kenma R, Yasumoto T (1987) Fluorimetric detection of diarrhetic shellfish toxins by high-performance liquid chromatograpy. Agric Biol Chem 51. $877-881$

Marcaillou-Le Baut C, Bardin B, Bardouil $M$, Bohec $M$, Le Dean L, Masselin P. Truquet P (1993) DSP depuration rates of mussels reared in a laboratory and an aquaculture pond. In: Smayda TJ, Shimizu Y (eds) Toxic phytoplankton blooms in the sea. Elsevier, Amsterdam, p 531-535

Marr IC, Hu T, Pleasance S, Quilliam M, Wricht J (1992) Detection of new 7-O-acyl-derivatives of diarthetic shellfish poisoning toxins by liquid chromatography-mass spectrometry. Toxicon 30:381-387

Murata M, Shimatani M, Sugitani H, Oshima $Y$, Yasumoto I (1982) Isolation and structural elucidation of the causative

Editorial responsibility: Otto Kinne (Editor),

Oldendorf/Luhe, Germany toxin of diarrhetic shellfish poisoning. Bull Jpn Soc Sci Fish 43:549-552

Poletti R, Viviani R, Casadei C, Lucentini L, Giannetti L, Funari E, Draisci $R$ (1996) Decontamination dynamics of mussels naturally contaminated with diarrhetic toxins relocated to a basin of the Adriatic Sea. In: Yasumoto T, Oshima Y, Fukuyo Y (eds) Harmful and toxic algal blooms. IOC of UNESCO, Paris, p 429-432

Riisgárd HU (1991) Filtration rate and growth in the blue mussel. Mytilus edulis Linnaeus, 1758: dependence on algal concentration. J Shellfish Res 10:29-35

Sampayo MA, Alvito P, Franca S, Sousa I (1990) Dinophysis spp. toxicity and relation to accompanying species. In: Granéli E, Sundström B, Edler L, Anderson DMM (eds) Toxic marine phytoplankton. Elsevier, New York, p $215-220$

Silvert W, Cembella, AD (1995) Dynamic modelling of phycotoxin kinetics in the blue mussel, Mytilus edulis, with implications for other marine invertebrates. Can J Fish Aquat Sci 52:521-521

Shumway SE, Cembella AD (1993) The impact of toxic algae on scallop culture and fisheries. Rev Fish Aquat Sci 1 $121-150$

Suganuma $M$, Suttajit $M$, Suguri $H$, Ojika $M$, Yamada $K$, Fujiki $H$ (1989) Specific binding of okadaic acid, a new tumour promoter in mouse skin. FEBS Lett 250:615-618

Wohlgeschaffen GD, Mann KH, Subba Rao DV, Pocklington R (1992) Dynamics of the phycotoxin domoic acid: accumulation and excretion in two commercially important bivalves. J Appl Phycol 4:297-310

Yasumoto T, Oshima Y, Yamaguchi M (1978) Occurrence of a new type of toxic shellfish in Japan and chemical properties of the toxin. Bull Jpn Soc Sci Fish 44:1249-1255

Yasumoto T, Murata M, Oshima Y, Sano M. Matsumoto, GK Clardy J (1985) Diarrhetic shellfish toxins. Tetrahedron 41: 1019-1025

Submitted: February 27, 1998; Accepted: September 24, 1997 Proofs received from author(s): January 11, 1999 